Check for updates

Cite this: RSC Adv., 2019, 9, 33931

Received 17th July 2019

Accepted 20th September 2019

DOI: $10.1039 / \mathrm{c} 9 \mathrm{ra05484g}$

rsc.li/rsc-advances

\section{Solvent assisted size effect on AuNPs and significant inhibition on K562 cells $\uparrow$}

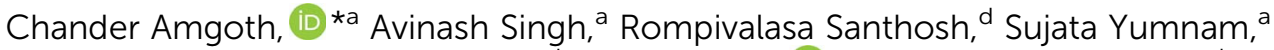 \\ Priyanka Mangla, ${ }^{a}$ Rajendra Karthik, ${ }^{b}$ Tang Guping (D) ${ }^{* c}$ and Murali Banavoth ${ }^{\star d}$
}

Herein, the synthesis and characterization of ideal size ( 10 and $40 \mathrm{~nm}$, in diameter) AuNPs (gold nanoparticles) were reported. Two different organic solvents such as DMF (dimethyl formamide) and NMPL (N-methyl-2-pyrrolidone) were used to synthesize AuNPs along with agents reducing agents such as $\mathrm{NaBH}_{4}$ (sodium borohydrate) and $\mathrm{Na}_{3} \mathrm{C}_{6} \mathrm{H}_{5} \mathrm{O}_{7}$ (sodium citrate). The combination of $\left[\left(\mathrm{HAuCl}_{4}\right)-(\mathrm{DMF})-\right.$ $\left(\mathrm{NaBH}_{4}\right)$ ] gives AuNPs with an avg. size of $10.2 \mathrm{~nm}$. Similarly, the combination of [(HAuCl 4$)-(\mathrm{NMPL})-$ $\left.\left(\mathrm{Na}_{3} \mathrm{C}_{6} \mathrm{H}_{5} \mathrm{O}_{7}\right)\right]$ gives AuNPs with an avg. size of $40.4 \mathrm{~nm}$. The morphology of these nanoscale AuNPs has been characterized through TEM and HRTEM imaging followed by SAED for lattice parameters such as $d$-spacing value $(2.6 \AA / 0.26 \mathrm{~nm}$ ) of crystalline metal (Au) nanoparticles. Further, these unique and ideal nanoscale AuNPs were used to evaluate the potential working efficacy by using in vitro cell based studies on K562 (leukaemia) blood cancer cells. From the MTT assay results around $88 \%$ cell inhibition was measured for $\sim 10 \mathrm{~nm}$ sized AuNPs. The treated cells were stained with different fluorescent dyes such as FITC, DAPI, Rho-6G and their ruptured morphology has been reported in the respective sections. These types of ideal sized metal (Au) nanoparticles are recommended for various theranostics such as to cure breast, colon, lung and liver cancers.

\section{Introduction}

There is the possibility to stabilize smaller sized nanoparticles through the increased polarity of solvents. ${ }^{1}$ Solvents stabilize the system through charge stabilization and capping approaches. As per the classical homogeneous nucleation theory, the homogeneous nucleation leads to growth of the particles spontaneously with the solvent effect. ${ }^{1,2}$ As per the supersaturation concept, the ionic product divided by solubility product helps in the homogeneous nucleation of AuNPs. The decrease in the solubility of an ionic material with non-polar solvent will show an adverse affect on the size of AuNPs followed by the heterogeneous nucleation. And this can be avoided by choosing the appropriate polar solvent for homogeneous, consistent nucleation for metal (Au) nanoparticles. ${ }^{2,3}$ With this chemical precipitation method, there is a high chance to get homogeneous nuclei followed by consistent sized particles and this happens because of the solvent and its concentration. Such

a Department of Science and Humanities, MLR Institute of Technology, Hyderabad-500043, TS, India

${ }^{b}$ Department of Electronics and Communication Engineering, MLR Institute of Technology, Hyderabad-500043, TS, India

${ }^{c}$ School of Chemistry, Zhejiang University, Hangzhou-310028, China ${ }^{d}$ School of Chemistry, University of Hyderabad, Hyderabad-500046, TS, India

$\dagger$ Electronic supplementary information (ESI) available. See DOI: 10.1039/c9ra05484g small AuNPs and the solvent effect has been applied for novel metal nanoparticles with various applications. ${ }^{\mathbf{1 - 4}}$

The oxalate based solvents lead to the single crystal formation for smaller nanoparticles but aggregation and agglomeration phenomena can rely on the chemical precipitation and show the adverse effect to enhance the particle sizes. ${ }^{5}$ The RSS (relative supersaturation) concept gives some important information to control the nucleation and crystal growth followed by the chemical precipitation methods. ${ }^{5,6}$ The different concentrations of reagents and solvents do show the adverse affect followed by the capping of metal (Au) nanoparticles. Sometimes atmospheric conditions such as temperature, $\mathrm{pH}$, stirring methods also show the effect on the size of particles. ${ }^{7}$ The redox (oxidation and reduction) reaction of $\mathrm{HAuCl}_{4}$ (tetrachloroauric acid) with $\mathrm{MoS}_{2}$ (molybdenum disulfide) leads to the formation of [(AuNPs)-( $\left.\left.\mathrm{MoS}_{2}\right)\right]$ nanocomposite. ${ }^{5-8}$ The organic solvent DMF (dimethyl formamide) shows higher rate of reduction on gold materials such as $\mathrm{Au}^{3+}$ (gold III) which gives smaller sized particles. ${ }^{\mathbf{1 , 8}, 9}$ However, organic solvent NMPL $(N$ methyl-2-pyrrolidone) has the lower reduction rate on metal/gold nanoparticles and it leads to the larger sized particles. ${ }^{\mathbf{1 0 , 1 1}}$ From the DMF and NMPL organic solvents it is evidenced that there is a chance to synthesize the gold nanoparticles in the size range of 8 to $40 \mathrm{~nm}$ (in diameter). With these organic solvents the stepwise increase in the size of metal (Au) nanoparticles can be achieved. Gold nanoparticles (AuNPs) have been under wide range of utilization since last couple of decade, especially industrial and pharmaceutical/biomedical fields. ${ }^{\mathbf{1 9 - 1 2}}$ 
Recently, these ideal sized metal (Au) nanoparticles have been used in various technological and biomedical applications such as organic photovoltaic, ${ }^{9}$ drug delivery systems (DDSs), ${ }^{10}$ sensory probes, ${ }^{\mathbf{1 1}, \mathbf{1 2}}$ pharmaceutical and therapeutic applications, catalysis and electronic conductors. ${ }^{\mathbf{1 1 - 1 4}}$ The size, shape and morphology/geometry of gold nanoparticles are changeable by tuning their physical and chemical properties. ${ }^{\mathbf{1 - 5 , 1 5}}$ These polar solvents propagate the interactions between the solvent molecules and metal particles to reduce the size and change the surface chemistry of nanoparticles through the aggregation and this aggregation can be avoided through the sonication methods. The monodispersed AuNPs with size range of 8$40 \mathrm{~nm}$ are most suitable for killing and to inhibit the proliferation of cancer cells. ${ }^{16}$ The concept surface plasmon resonance can help to kill the cancer cells predominantly through the dosage based localization inside the cells. ${ }^{16,17}$ These ideal sized AuNPs are most suitable tools to penetrate the cell membrane and to show the killing and inhibition activity significantly. The concept surface plasmon resonance depends on the size of metal $(\mathrm{Au})$ nanoparticles and it can be applied for various theranostics. Interestingly, these gold nanoparticles (AuNPs) have been used for extensive studies based on their colloidal stability, surface chemistry, capping nature, surface tunability based on its chemical and physical properties. All these properties will do change from bulk to nanoscale sizes. ${ }^{18}$ Particularly, the AuNPs with the size range of $10-40 \mathrm{~nm}$ are of great interest due to the interface science between bulk to nanoscale reduction followed by the molecular regime, and interactions between solvent to metal (Au) nanoparticles. Similarly, these synthesized small sized (10-40 nm) AuNPs are of great interest because of their catalytic properties followed by the easy penetration and incorporation inside the cancer cells and other theranostics. ${ }^{15-19}$

Furthermore, the recent nanotechnological advances in the biomedical field has shown potential working efficacy of these 10-40 nm sized AuNPs can be utilized as contrast agents for blood pool assay and CT (computed tomography) scanning such as tumour computed tomography imaging etc. These nanosized AuNPs are ideal and unique in their activities to enhance the image contrast while CT scanning followed by the in vivo animal model imaging. These AuNPs not only used in CT scanning, they can be used in PDT (photodynamic theory) for various treatments and surgeries based on their ideal particle size suitability and changeability. The ideal sized (10-40 nm) AuNPs are recommended for hyperthermia therapy to kill the cancer cells through the rapid heating of gold nanoparticles and interactions with the cancer cells lead to rupture the cells. ${ }^{20}$ However, these AuNPs can be coated or loaded with the nanomedicines and anticancer drugs and referred as nanoformulations as therapeutic agents for drug delivery systems (DDSs) to treat and cure various diseases and ailments. The synthesis and characterization of nanosized gold particles (AuNPs) are used in various fields such as therapeutic agents, sensory applications, nanobiotechnology, catalysis, calorimetric applications, MRI and CT scanning, to know the vibrational and chemical bonding energies and strengths, detection of proteins, genetic material, pollutants and other industrial and pharmaceutical applications. ${ }^{17-22}$ These nanoscale AuNPs can be used for many diagnostic methods such as detection of biomarkers, heart diseases, lung, liver, prostate, colon cancers and other infectious diseases. The AuNPs (gold nanoparticle) surface can be used as selective oxidation followed by the surface modifications for various fuel cell and automotive applications. ${ }^{21,23,24,29}$

\section{Materials and methods}

\subsection{Materials}

All the chemicals required for the synthesis of nanoscale AuNPs has been purchased with high purity and used without further purification. The $\mathrm{HAuCl}_{4}$ (tetrachloroauric acid, 98.9\%, Sigma Aldrich), DMF (dimethyl formamide, 97.6\%, Sigma Aldrich), $\mathrm{NaBH}_{4}$ (sodium borohydrate, $>97.8 \%$, SDFCL), NMPL ( $N$ methyl-2-pyrrolidone, $>98 \%$, Sigma Aldrich), $\mathrm{Na}_{3} \mathrm{C}_{6} \mathrm{H}_{5} \mathrm{O}_{7}$ (sodium citrate, $>98 \%$, SDFCL), IPA (isopropanol, $>98 \%$, SDFCL), methanol ( $>96 \%$, SDFCL), acetone extra pure $(>96 \%$, sigma Aldrich), carbon coated copper grids with 200 mesh size (Ted Pella Inc), MTT (methyl thiazol tetrazolium, >99.9\%, Sigma Aldrich), magnetic bead, magnetic stirrer cum hot plate, sample viols, micropipettes and centrifuge tubes. Apart from these chemicals, various other glassware, consumables such as beakers, stirrer, magnetic bead, hot plate, goggles, gloves etc.

\subsection{Synthesis of AuNPs with an avg. size of $10 \mathrm{~nm}$}

The chemical compound $\mathrm{HAuCl}_{4}$ (tetrachloroaurate/auric acid) of $\sim 2.5 \mathrm{~g}$ was taken in a $250 \mathrm{~mL} \mathrm{RB}$ flask (round bottom flask). This has been added with $\sim 60 \mathrm{~mL}$ of organic solvent DMF (dimethyl formamide) and allowed to stir for $12 \mathrm{~h}$ at $50{ }^{\circ} \mathrm{C}$. This is again added with the $0.5 \mathrm{~g}$ of $\mathrm{NaBH}_{4}$ (sodium borohydrate) strong reducing agent and allowed to stir for $2 \mathrm{~h}$ at $50{ }^{\circ} \mathrm{C}$. Furthermore, to that mixture methanol $(\mathrm{MeOH}) \sim 25 \mathrm{~mL}$ has been added and allowed to stir for $2 \mathrm{~h}$ at $50{ }^{\circ} \mathrm{C} .^{1,11,17,25}$ Finally, the sodium metal from reducing agent $\mathrm{NaBH}_{4}$ was degraded with the methanol solvent. The end product of reaction was yellowish in colour and this solution was used to separate the metal (Au) nanoparticles through the high speed centrifugation at 10000 RPM for 10 minutes. The final gold nanoparticles were dispersed in IPA (isopropanol) solution and used to prepare the samples for TEM, HRTEM and SAED characterizations. The physical appearance of final gold sample has been reported in the inset of the Fig. $6 \mathrm{a}$ and its respective dispersion of gold nanoparticles with TEM micrographic imaging. The yield of the final synthesized nanoscale AuNPs is calculated as $1.7 / 2.5 \mathrm{~g}$ (w/ $\mathrm{w} \%$ ) which is equal to $\sim 68 \%$.

\subsection{Synthesis of AuNPs with an avg. size of $40 \mathrm{~nm}$}

The chemical compound $\mathrm{HAuCl}_{4}$ (tetrachloroaurate/auric acid) of $\sim 2.5 \mathrm{~g}$ was taken in a $250 \mathrm{~mL}$ RB flask (round bottom flask). This has been added with $\sim 60 \mathrm{~mL}$ of organic solvent NMPL $(N-$ methyl-2-pyrrolidone) and allowed to stir for $12 \mathrm{~h}$ at $50{ }^{\circ} \mathrm{C}$. It is again added with the $0.5 \mathrm{~g}$ of $\mathrm{Na}_{3} \mathrm{C}_{6} \mathrm{H}_{5} \mathrm{O}_{7}$ (sodium citrate, $>98 \%$, SDFCL), strong reducing agent and allowed to stir for $2 \mathrm{~h}$ at $50{ }^{\circ} \mathrm{C}$. Furthermore, to that mixture $\sim 25 \mathrm{~mL}$ of methanol 
$(\mathrm{MeOH})$ has been added and allowed to stir for $2 \mathrm{~h}$ at $50{ }^{\circ} \mathrm{C}$. Finally, the sodium metal from reducing agent $\mathrm{Na}_{3} \mathrm{C}_{6} \mathrm{H}_{5} \mathrm{O}_{7}$ was degraded with the methanol solvent. The end product of reaction was dark red in colour and this solution was used to separate the metal (Au) nanoparticles through the high speed centrifugation at 10000 RPM for 10 minutes.,11,17,26-29 The final AuNPs were dispersed in IPA (isopropanol) solution and used to prepare the samples for TEM, HRTEM and SAED characterizations. The physical appearance of final gold sample has been reported in the inset of the Fig. $6 \mathrm{~b}$ and its respective dispersion of gold nanoparticles with TEM micrographic imaging. The yield of the final synthesized nanoscale AuNPs is calculated as $2.0 / 2.5 \mathrm{~g}(\mathrm{w} / \mathrm{w} \%)$ which is equal to $\sim 80 \%$.

\subsection{Sample preparation for TEM imaging}

The synthesized AuNPs of $0.2 \mathrm{~g}$ was dispersed in $3 \mathrm{~mL}$ of IPA (isopropanol) solution and used for the sample preparation for TEM, HRTEM and SAED characterizations. The dispersion followed by the ultra sonication of AuNPs with the help of isopropanol solvent. Then, the $\sim 20 \mu \mathrm{L}$ of well dispersed solution of AuNPs was drop casted on the surface of the carbon coated copper grids with the help of micropipette and grids were allowed to dry for over knight. The well dried samples were used for TEM imaging at $200 \mathrm{kV}$ accelerating voltage. .11,17,19,30 $^{\text {These }}$ crystalline metal $\mathrm{(Au}$ ) nanoparticles does not show any charge effect at such higher accelerating voltages because of their high melting point and its crystalline nature.

\section{Results and discussions}

\subsection{Morphology of AuNPs}

The Fig. 1 schematic illustrate the steps involved in the synthesis of nanoscale AuNPs ${ }^{31}$ followed by the required solvents, reducing agents and source of gold nanoparticles and experimental procedures. The synthesis of ideal sized nanoscale gold nanoparticles (AuNPs) with changeable size, shape, and morphology and surface properties is a unique and novel approach for the scientific research community. ${ }^{28-32}$ The spherical shaped gold nanoparticles are scientifically useful for the many industrial and pharmaceutical applications. This can

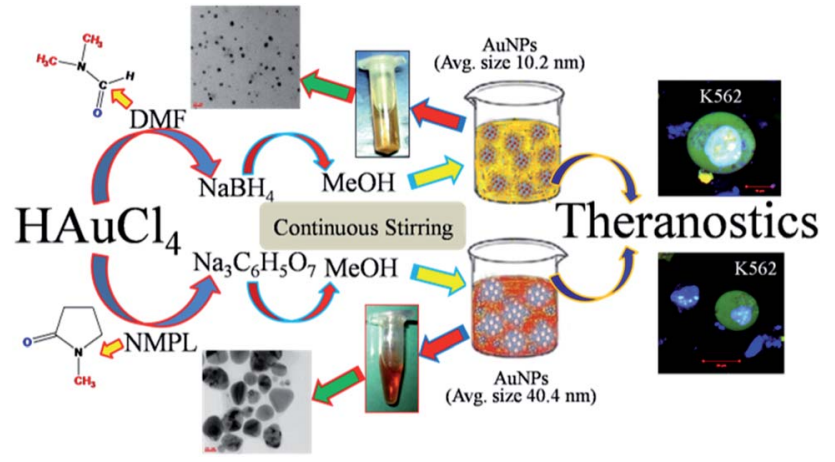

Fig. 1 Schematic illustrate the steps involved in the synthesis of nanoscale AuNPs followed by the required solvents, reducing agents and source of gold nanoparticles and experimental procedures. be achieved through the selection of solvents, reducing agents, concentration, temperature, $\mathrm{pH}$ of the system, sonication methods etc. The organic solvents dimethyl formamide (DMF) and $N$-methyl-2-pyrrolidone (NMPL) has the role in reduction of the size of the gold nanoparticles along with the reducing agents such as sodium citrate $\left(\mathrm{Na}_{3} \mathrm{C}_{6} \mathrm{H}_{5} \mathrm{O}_{7}\right)$ and sodium borohydride $\left(\mathrm{NaBH}_{4}\right)$. The solvent methanol $(\mathrm{MeOH})$ has the property to degrade and decompose the sodium ( $\mathrm{Na}$ ) metal to get the ideal nanoscale particles. The Fig. S1 and $\mathrm{S} 2 \uparrow$ correspond to TEM micrographs of AuNPs synthesized from DMF and $\mathrm{NaBH}_{4}$. These images were acquired from lower to higher magnification and shows ideal nanosized gold particles.

The Fig. 2a-d corresponds to the TEM micrographs acquired from lower to higher magnification such as scale bar of 100, 50, 20 and $5 \mathrm{~nm}$ respectively. As the magnification increases, the depth of focus (DOF) decreases and it leads to reduce the scanning area with that less number of nanoparticles can be seen in higher magnified images and the Fig. $2 \mathrm{~d}$ has very few nanoparticle at higher magnification. These are smaller gold nanoparticles with an average particle size of $10.4 \mathrm{~nm} .^{31-33}$

These ideal nanosized gold nanoparticles (AuNPs) were synthesized by using the DMF organic solvent along with strong reducing agent sodium borohydrate (detailed synthesis mentioned in the Section 2.2 of materials and methods). The $\mathrm{HAuCl}_{4}$ has been used as source precursor materials for gold nanoparticles and reaction was performed in the methanol solvent at $50{ }^{\circ} \mathrm{C}$ for several hours. From the Fig. 2a-d TEM micrographs, the monodispersed and well distributed AuNPs are observed. The surface chemistry of AuNPs depends on the solvent and concentration of solvent followed by the formation of capping agents on the surface of the nanoparticles. In this case, AuNPs size is almost consistent with monodispersion of

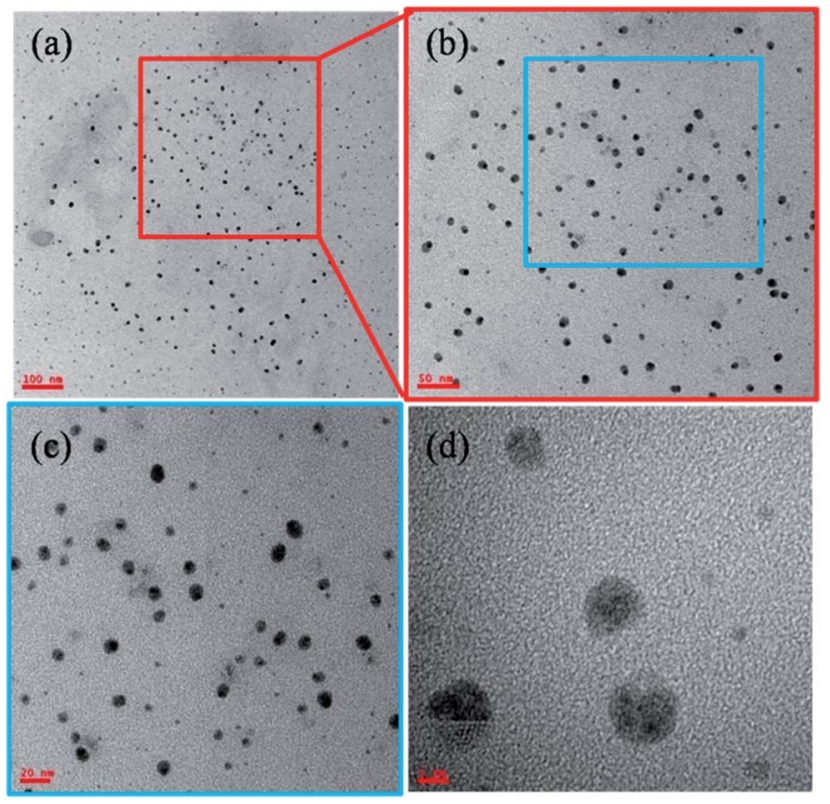

Fig. 2 TEM micrographs of smaller (avg. particle size is $10.4 \mathrm{~nm}$ ), gold nanoparticles (AuNPs). The images $(a-d)$ are acquired from lower to higher magnification. 
particles throughout the sample or TEM grid surface. ${ }^{29-34}$ These AuNPs are spherical shaped with insistent size and dispersion throughout the surface and it rely on the reducing agents used during the synthesis process. The harsh reducing agent encourages the heterogeneous nucleation and it lead to give highly inconsistent size, shape and irregular dispersion of nanoparticles. The sonication methods, temperature of the sonication bath, sonication time will also affect the consistency in getting the uniform sized nanoparticles. The Fig. 3a and b corresponds to the AuNPs captured from lower to higher magnification. The Fig. 3 a has been acquired at lower magnification and it shows large number of AuNPs.

\subsection{Interplanar distance and solvent effect on AuNPs}

The Fig. 3b acquired at higher magnification and only one nanoparticle has been seen at such higher resolution. It represents the lattice fringes and this image has been used to calculate the lattice parameters such as $d$-spacing (Fig. 3c). To get these $d$-spacing values, the raw data has been imported to Gatan software and used bright spot method to analyze the lattice fringes (inter-planar distances) as reported in the Fig. 3c and it gives $2.6 \AA / 0.26 \mathrm{~nm}$ which is highlighted in the figure. This has been evidenced by the SAED (selected area electron diffraction) in the Fig. $3 \mathrm{~d}$ and this $d$-spacing value has been measured through the two opposite bright spots of the same circle. ${ }^{1,11,17,19,30-35}$

Further, this $d$-spacing (inter-planar distance) value (0.26 $\mathrm{nm}$ ) has been confirmed by suing the IFFT method (inset in Fig. 3d) and its corresponding values. Interestingly, these TEM characterization studies disclose the uniqueness of physical and chemical properties dependency on polarity of solvent as
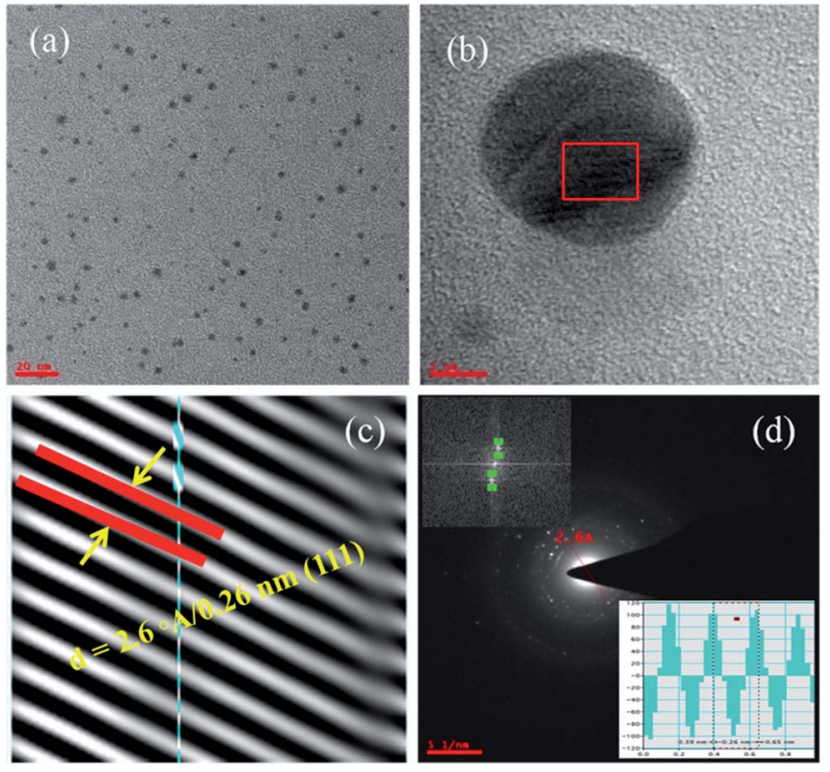

Fig. 3 TEM micrographs ( $a$ and b) for AuNPs are acquired from lower to higher magnifications. The image (c) corresponds to AuNPs lattice fringes which corroborates the $d$-spacing value of $0.26 \mathrm{~nm}$ and image (d) corresponds to SAED for AuNPs and its IFFT (inset) evidences the $d$ spacing value. well nature of reducing agents with respect to reaction methods and conditions. The size, shape and morphology followed by the smooth and rough surface of the gold nanoparticles is extensively dependent on the variable parameters such as temperature $(T)$, volume $(V)$, number of moles $(n)$, pressure $(P)$ of reaction chamber etc. From the TEM micrographs (Fig. 2 and 3), it has been confirmed that the synthesized AuNPs are not capped or covered by any residues which means surface of the particles is smooth with spherical shaped morphology. The most important strategy involved in the synthesis of ideal sized AuNPs is dependent on the selection of organic solvent followed by the Schiffrin-Brust approach. This strategy helps in the design and development of AuNPs with tunable size, shape and morphology. This methodology shows significant effect on the size, shape and morphology through the homogeneous nucleation followed by the monodispersion of nanoparticles and consistent size of particles.

However, the crystalline metal (AuNPs) are stable at $200 \mathrm{kV}$ accelerating voltages while TEM imaging. Our main aim is to design and develop ideal sized AuNPs which are in the size range of $\leq 50 \mathrm{~nm}$ and it is achieved through the modified Schiffrin-Brust approach by using organic solvents and strongreducing agents. The Fig. $\mathrm{S} 3 \uparrow$ corresponds to TEM images of AuNPs synthesized by using NMPL organic solvent and sodium citrate $\left(\mathrm{Na}_{3} \mathrm{C}_{6} \mathrm{H}_{5} \mathrm{O}_{7}\right)$ which results in the large sized nanoparticles. ${ }^{1-6,17-23,35}$

The Fig. 4a-d TEM micrographs are acquired from lower to higher magnification and these are synthesized by using the NMPL organic solvent and sodium citrate as strong reducing agent (detailed synthesis mentioned in the Section 2.3 of materials and methods). From these TEM images, the irregular shaped AuNPs are observed with highly inconsistent size of

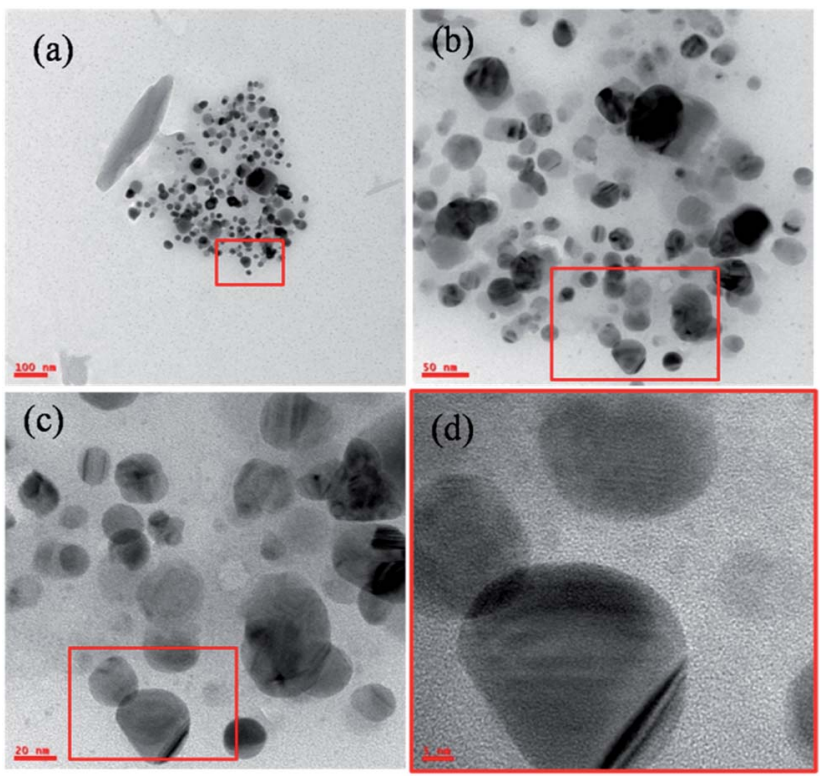

Fig. 4 TEM micrographs for larger AuNPs (avg. particle size is 40.2 $\mathrm{nm})$. The images $(\mathrm{a}-\mathrm{d})$ are acquired from lower to higher magnification. From the image (d) the lattice fringes can be observed. 
particles. From the Fig. 4b, huge irregularity and inconsistency of AuNPs has been seen. The dispersion and distribution of nanoparticles are interpreted through these TEM images. The highly magnified Fig. 4d corroborates the morphology and lattice fringes of crystalline metal $(\mathrm{Au})$ nanoparticles. The more inconsistency in the size and shape of these AuNPs is due to the heterogeneous nucleation of gold nanoparticles controlled by the NMPL organic solvent and reducing agent (sodium citrate). The hexagonal morphology of gold nanoparticles has been observed through these TEM images and this represents the well segregated nanoparticles without any surface defects. The AuNPs basically show phase changes with organic solvents but here it is quantitatively not showing any adverse affect on the size, shape and morphology of nanoparticles. The increased size in this case is due to the lower affect of organic solvent and reducing agent too. The polarity of the used organic solvent shows adverse affect on the increase and decrease of size of metal (Au) nanoparticle sizes. Here in this case, do note that the sodium citrate (reducing agent) has lower affect and it helps in increase of the particle size from 10-40 nm. However, these AuNPs are unique in their size, shape and morphology which are dependent on the reactions conditions. The samples in the Fig. 1 schematic representation shows light yellow colour for 8$12 \mathrm{~nm}$ sized AuNPs and dark red for the 30-40 nm. The Fig. S4 $\dagger$ corresponds to EDAX and it represents the elemental composition for synthesized AuNPs. Through the EDAX analysis, the following elements were observed in the sample which results in the presence of oxygen (O) with 55.44 (weight\%) and 93.87 (atomic\%) is because of impurities present in the sample. The inset table shows the atomic (6.13) and weight (44.56) percentages of $\mathrm{Au}$ element. There are high chances to get the elemental composition for $\mathrm{Cl}$ (chlorine), W (tungsten), Ag (silver), Nb (niobium) because of impurities present in the sample followed by the external contaminations.

\subsection{Particle size analysis}

The crystalline metal ( $\mathrm{Au}$ ) nanoparticles are hexagonal shaped with cubic close packing or FCC (face cantered cubic) crystal lattice structure. From the Fig. $4 \mathrm{~b}$ and 5b, the HRTEM images inter-planar distances are visible but those cannot be differentiated. In such cases Fig. $4 \mathrm{c}$ and $5 \mathrm{c}$ for both the (smaller and larger) nanoparticles were analyzed through the $\mathrm{DM}_{3}$ data files and enlarged inter-planar distances are reported as $2.6 \AA(0.26$ $\mathrm{nm}$ ) corresponds to the (111) crystal plane which is consistent value for both the AuNPs samples. The organic polar environment is the main factor to affect or reduce the AuNPs size along with strong reducing agents. ${ }^{\mathbf{1 4 , 1 9 - 2 8 , 3 6}}$ These are showing significant influence on controlling the metal nanoparticle size, shape and morphology through the self-assembly and affinity between metal atoms and polar solvents with the help of reducing agents as size changing agents. The Fig. 5a and $\mathrm{b}$ acquired from lower to higher magnification for the larger AuNPs (scale bar 20 to $5 \mathrm{~nm}$ ) and we can see more number of particles at lower magnification with triangular, hexagonal, spherical and even more irregular shaped gold nanoparticles. From the Fig. 5d, once again the inter-planar distances (2.6
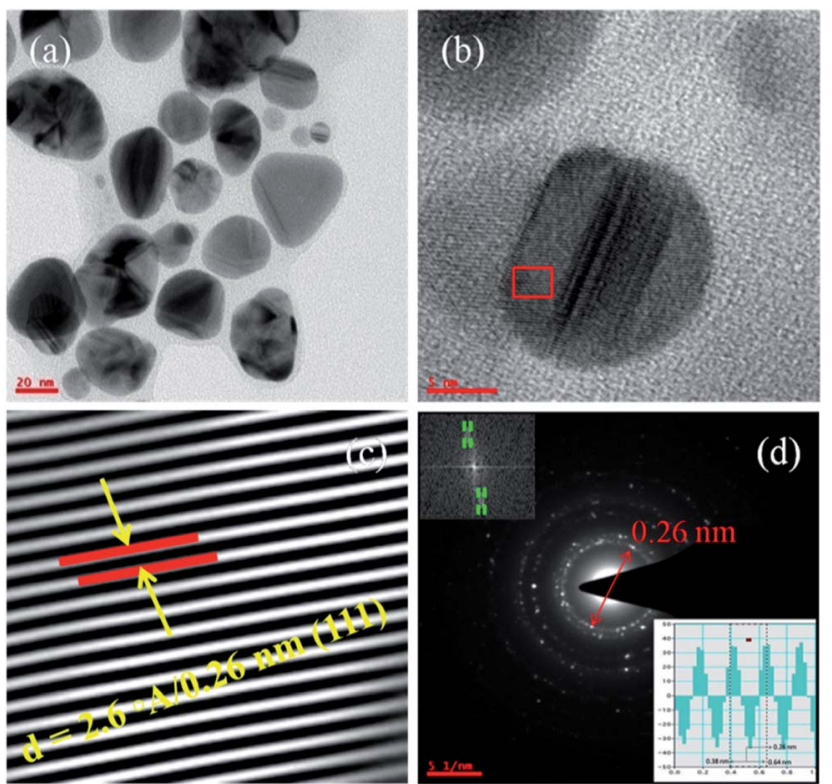

Fig. 5 TEM micrographs ( $a$ and b) for AuNPs are acquired from lower to higher magnifications. The image (c) corresponds to AuNPs lattice fringes which corroborates the $d$-spacing value of $0.26 \mathrm{~nm}$ for larger nanoparticles. The image (d) corresponds to SAED for AuNPs and its IFFT (inset) which evidences the $d$-spacing value.

$\AA$ A/0.26 nm with (111) plane) for the Au metal with FCC crystal system has been evidenced. The inset in Fig. 5d, the IFFT data also evidences the lattice fringes for crystalline metal (Au) nanosystem. It also illustrate the (inset) of two bright spots joined for interplaner distances of AuNPs. ${ }^{27,28,36}$

The Fig. $6 \mathrm{a}$ and $\mathrm{b}$ corresponds to particle size distribution for the AuNPs synthesized in both the approaches. The Fig. 6a corroborates the particle size distribution for smaller AuNPs whose average particle size is $10.4 \mathrm{~nm}$. The inset sample vial represents the physical appearance (light yellow colour) of these nanoscale gold nanoparticles. Furthermore, inset TEM micrograph illustrates the diameter of particles marked while imaging and size, shape and morphology of synthesized AuNPs with their distribution and dispersion.

The particle size distribution has been calculated through the free hand polygon method which interprets the count of number of particles followed by the size distribution in diameter. The inset table corroborates the parameters involved in the non-linear Gauss curve fit. The regression coefficient $\left(R^{2}\right)$ value for the distribution plot has been obtained as 0.9192 which signifies the best non-linear curve fit after 100 iterations. The FWHM (full width half maxima) value is 7.86 and height value as 20.52 and all other parameter were also incorporated in the respective table. Similarly, the Fig. 6b corroborates the particle size distribution for larger AuNPs. The physical appearance (dark red colour) of these particles can be seen in the sample vial incorporated in the inset of the figure. The inset TEM micrograph illustrates the size, shape and morphology of larger nanoparticles. Some of the particles are marked with their diameter values through the TEM characterizations. The plotted bar graphs represents the average size of AuNPs is $40.2 \mathrm{~nm}$ in 

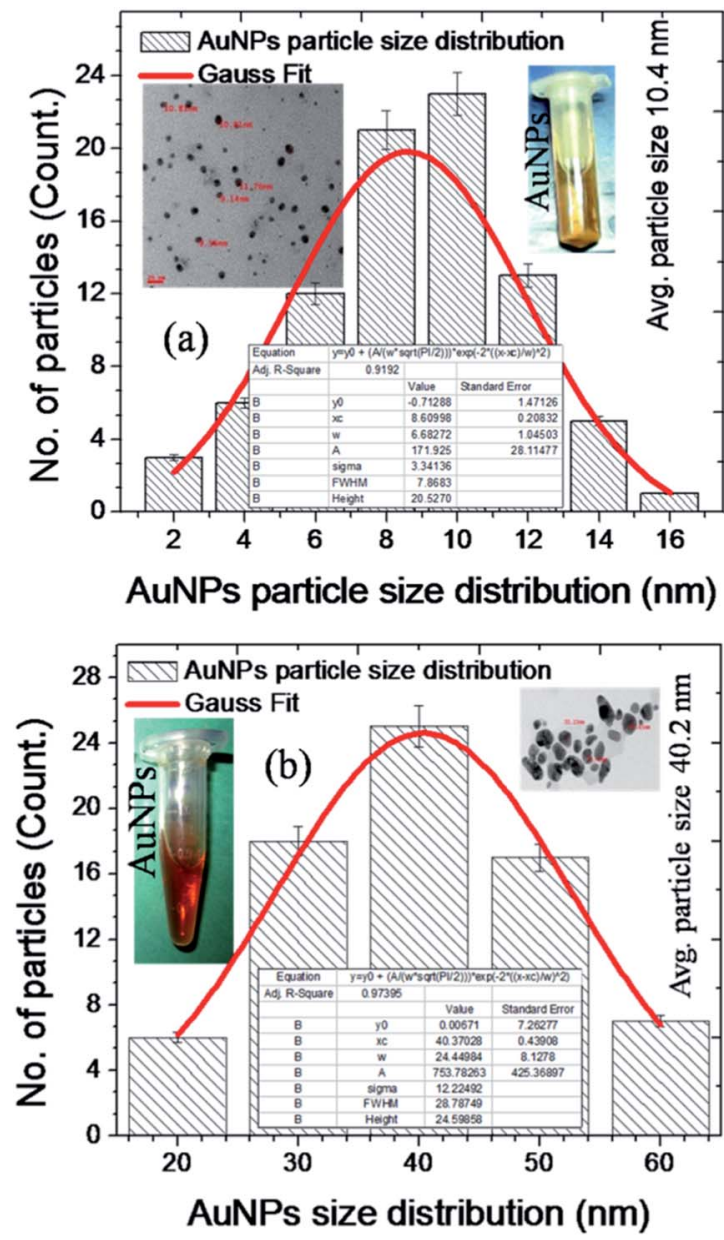

Fig. 6 Plot ( $a$ and $b$ ) shows the AuNPs particles size distribution for smaller $(\sim 10 \mathrm{~nm})$ and larger ( $\sim 40 \mathrm{~nm})$ sized nanoparticles.

diameter. The inserted table illustrates the values and parameters for the non-linear Gauss curve fit for larger AuNPs. The $R$ square $\left(R^{2}\right)$ or regression coefficient value for the Gauss fit has been calculated as 0.9739 after 100 iterations and it represents the best curve fit for the large scale sized AuNPs. Furthermore, the FWHM value is calculated as 28.78 , sigma value as 12.22 and height value as 24.59 were imbedded in the respective table to easy understanding of all the AuNPs properties and parameters. The AuNPs in the size range of $60-80 \mathrm{~nm}$ in diameter can be achieved through the aqueous and non-polar organic solvents. The ideal sized (particle size 8-12 nm) nanoparticles cannot be extracted through the polar organic solvents with strong reducing agents. The AuNPs in the size range of $8-12$ are especially attractive for many biomedical and pharmaceutical applications. Herein, these ideal nanoscale AuNPs were synthesized without using any surfactants. As per the literature AuNPs beyond a certain size $(>50 \mathrm{~nm})$ are prone to flocculation (coagulation and agglomeration) because of van der Waals's interactions to stabilize the particles. Here nanoscale AuNPs do not show any flocculation and this is happened due to absence of surfactants. ${ }^{1-8,11,17,27,28,37}$
The use of non-polar solvents such as $\mathrm{CHCl}_{3}$ (chloroform) and toluene results in the formation of AuNPs with size of $>80 \mathrm{~nm}$ followed by the flocculation in nanoparticles. The narrow sized gold nanoparticles have good monodispersion with normal solvents like methanol, ethanol, isopropanol, acetone etc. These ideal sized AuNPs are stable at room temperature (RT i.e. $\left.\sim 25^{\circ} \mathrm{C}\right)$ and body temperatures $\left(\sim 37.4^{\circ} \mathrm{C}\right)$. This surfactant free AuNPs are free from flocculation and used for various industrial and pharmaceutical fields due to their indefinite stability at RT and body temperatures. It is worth mentioning that the size or hydrodynamic volume of AuNPs can be increased upon blending with polymers such as PS (poly styrene), PCL (poly caprolactone) etc. The Table 1 represents the source materials for gold nanoparticles $\left(\mathrm{HAuCl}_{4}\right)$, organic solvents used for the reactions, reducing agents used during the synthesis of nanoscale AuNPs and average size of final AuNPs obtained through the our modified synthesis methods. .8,32,36,37 $^{2}$

From the TEM micrographs, it is disclosed that the AuNPs dispersions demonstrate the long-term stability of particles without any capping agents on the surface of the particles helps in easy penetration and insertion inside the infected cells of human body. Further, these ideal sized AuNPs were used to evaluate the working efficacy on K562 (leukaemia) blood cancer cells and results were incorporated in the following sections. ${ }^{1,28,32,36-38}$

The UV-visible absorbance spectrum (Fig. 7) corroborates the absorbance of two different sized gold nanoparticles. The smaller and ideal sized AuNPs of avg. Size $10.4 \mathrm{~nm}$ has the sharp peak compare to AuNPs of avg. Size $40.2 \mathrm{~nm}$. The highest and most significant peak for the synthesized AuNPs has been obtained at the wavelength $\left(\lambda_{\max }\right)$ of $\sim 408$ for both sized gold nanoparticles. Around $30 \mu \mathrm{L}$ of each sample has been used for UV-visible absorption spectrum. The inset in Fig. 7 represents the physical appearance of gold nanoparticle sample. The UVvisible absorbance for AuNPs samples were performed at different concentrations such as AuNPs-I at $30 \mu \mathrm{L}, 60 \mu \mathrm{L}, 90 \mu \mathrm{L}$, $120 \mu \mathrm{L}$, respectively. Similarly for AuNPs-II sample and spectra for $30 \mu \mathrm{L}$ of both the AuNPs-I and II has been appended. From the UV-visible spectrum, it has been observed that the peak intensity has been reduced with the increase of size of AuNPs. The shifting in peak position from $\sim 406 \mathrm{~nm}$ to $\sim 410 \mathrm{~nm}$ has been observed for both the 40 and $10 \mathrm{~nm}$ sized AuNPs.

\section{4 $\mathrm{IC}_{50}$ on $\mathrm{K562}$ cells and bacterial species}

The $\mathrm{IC}_{50}$ (minimum inhibitory concentration) of different size AuNPs on leukemia blood cancer cells (K562) has been performed and results were appended in the respective sections. The minimum inhibitory concentrations $\left(\mathrm{MIC} / \mathrm{IC}_{50}\right)$ for small $(10 \mathrm{~nm})$ sized gold nanoparticles has been obtained to be 0.404 $( \pm 0.022) \mu \mathrm{M}$. Whereas $\mathrm{IC}_{50}$ values for large $(40 \mathrm{~nm})$ sized gold nanoparticles has been obtained to be $0.198( \pm 0.32) \mu \mathrm{M}$. From these $\mathrm{IC}_{50}$ values it is concluded that small $(10 \mathrm{~nm})$ sized AuNPs are potential tools to penetrate the cancer cell (K562) membrane and shows enhanced activity to kill and rupture the cell.

Furthermore, minimum inhibitory concentration (MICs) of two types of gold nanoparticles was evaluated by using different 
Table 1 Illustrate the source of gold nanoparticles, organic solvents used for the synthesis of AuNPs followed by the reducing agents and average size of synthesized AuNPs

\begin{tabular}{lllll}
\hline S. no & Source of gold & Solvent & Reducing agent & Avg. size of AuNPs \\
\hline 1 & $\mathrm{HAuCl}_{4}$ & DMF (dimethyl formamide) & $\mathrm{NaBH}_{4}$ & $10.4 \mathrm{~nm}$ (Fig. 6a) \\
2 & $\mathrm{HAuCl}_{4}$ & NMPL ( $N$-methyl-2-pyrrolidone) & $\mathrm{Na}_{3} \mathrm{C}_{6} \mathrm{H}_{5} \mathrm{O}_{7}$ & $40.2 \mathrm{~nm}($ Fig. $6 \mathrm{~b})$
\end{tabular}

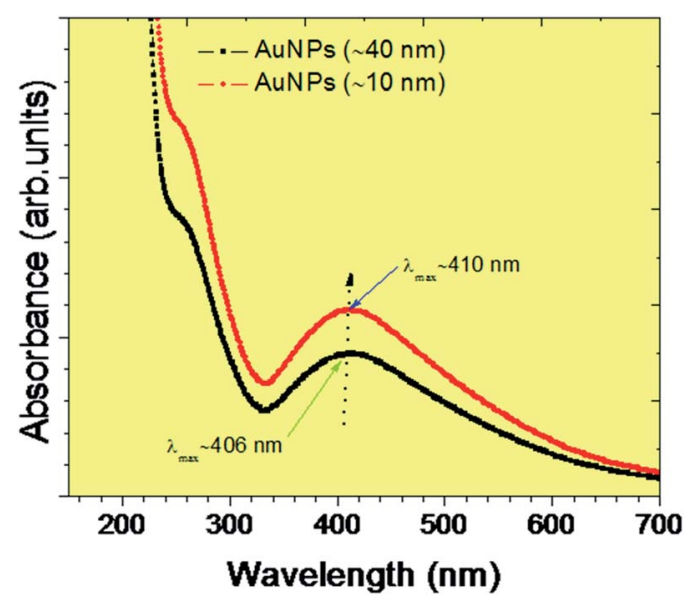

Fig. 7 Plot corroborate the UV-visible absorbance of two different sized gold (AuNPs) nanoparticles such as for $\sim 10$ and $40 \mathrm{~nm}$.

bacterial species such as Gram positive bacteria (Bacillus subtilis, Bacillus cereus, and Staphylococcus aureus) and Gram negative bacteria (Klebsiella pneumoniae, Proteus mirabilis, and Escherichia coli). All the bacterial species were grown and cultured in MullerHinton $(\mathrm{MH})$ broth growth medium with humidified atmospheric conditions maintained through the incubation at $\sim 37{ }^{\circ} \mathrm{C}$ over night. The inhibition on both the bacterial species has been appended in the following Table 2 . The smaller $(10 \mathrm{~nm})$ sized gold (AuNPs) shows grater inhibition on both the Gram positive and Gram negative bacterial species. The larger $(40 \mathrm{~nm})$ sized gold (AuNPs) shows lower inhibition compare to smaller AuNPs. This is also lower for K562 cancer cells too.

\subsection{Cell culture}

The synthesized AuNPs of $\sim 10 \mathrm{~nm}$ and $\sim 40 \mathrm{~nm}$ were further used to evaluate its working efficacy through the cell inhibition studies. Initially, K562 (leukaemia) blood cancer cells were cultured in $\mathrm{CO}_{2}$ incubator which is situated in a sterile culture room. However, the 6-well palates were used for the cell culture purpose and each well of the 6-well plate was added with RPMI media which is supplemented with $10 \%$ FBS (fetal bovine serum albumin), $100 \mathrm{IU} \mathrm{mL}^{-1}$ penicillin, $100 \mu \mathrm{g} \mathrm{mL}^{-1}$ streptomycin and $2 \mathrm{mM}$ L-glutamine. This RPMI medium helps in growing the $\mathrm{K} 562$ cells efficiently. They were maintained in a humidified atmosphere with $5 \%$ (volume percentage) of $\mathrm{CO}_{2}$ (carbon dioxide) at $\sim 37^{\circ} \mathrm{C}$. The cells were sub-cultured once in every 3 days. The density of around 50000 cells was taken for cell culture purpose. These cells were stored in liquid nitrogen temperatures $(77 \mathrm{~K})$. Finally, the well grown and cultured $\mathrm{K} 562$ cells were used for cell inhibition assay with combination of synthesized AuNPs. ${ }^{11,19,39}$

\subsection{Cell inhibition assay}

The cultured K562 cells were used for cell inhibition studies inassociation with synthesized $\sim 10$ and $40 \mathrm{~nm}$ AuNPs. A series of six (6) wells in a 96-well plate were chosen for cell inhibition assay. However, first well was marked as control or blank well which has been loaded with K562 cells as cultured, which means without any AuNPs. Rest five (5) wells of 96-well plate were poured with an approximate amount of $\sim 3 \times 10^{4}$ cells per well. These $\mathrm{K} 562$ cell containing wells were again added with $\sim 10 \mathrm{~nm}$ sized AuNPs whose concentration is calculated as $0.1 \mathrm{M}$ and each well was added with quantity of 200, 400, 600, 800 and $1000 \mathrm{ng} \mathrm{mL}^{-1}$ of AuNPs to each well of 96-well plate. ${ }^{38-41}$ The plate has been kept under slow agitation with 50 RPM for thorough mixing of K562 cells and $\sim 10 \mathrm{~nm}$ sized AuNPs. After thorough mixing, the 96-well plate was incubated in $\mathrm{CO}_{2}$ incubator for $36 \mathrm{~h}$ treatment between K562 cells and AuNPs with humidified temperature at $\sim 37{ }^{\circ} \mathrm{C}$. After $36 \mathrm{~h}$ treatment followed by the incubation of [(K562 cells $)-(\sim 10 \mathrm{~nm}$ sized AuNPs $)]$,

Table 2 Corroborate the MIC (minimum inhibitory concentration) of AuNPs on different bacterial species such as Gram positive and Gram negative

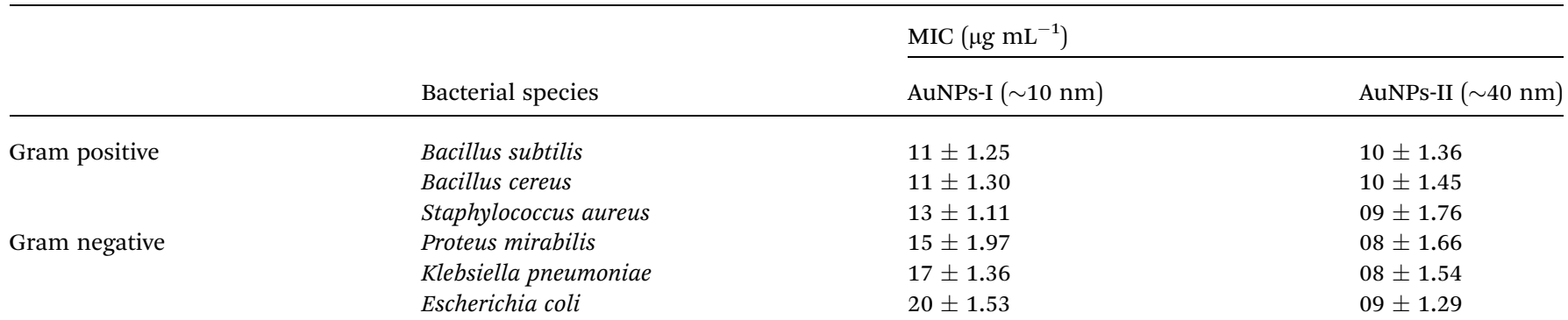


the 96-well plate was taken out from $\mathrm{CO}_{2}$ incubator and used to measure the cell inhibition (\%) followed by the MTT assay. ${ }^{\mathbf{4 2 , 4 3}}$ Further, the 96-well plate has been added with purple coloured MTT formazan chemical and plate used to measure the percentage of cell inhibition through the multi-plate reader (Biotek Synergy ${ }^{4}$ model). The Fig. S5 $\dagger$ bar graph illustrates the cell inhibition with small sized ( $10 \mathrm{~nm})$ AuNPs. The synthesized ideal sized $(\sim 10 \mathrm{~nm})$ gold nanoparticles were directly incubated with the cultured K562 cells and inhibition measurements performed after $36 \mathrm{~h}$ treatment. The cell inhibition (\%) for 200, 400, 600, 800 and $1000 \mathrm{ng} \mathrm{mL}^{-1}$ of quantities of $0.1 \mathrm{M}$ concentrated AuNPs shows 42, 58, 64, 78 and 88, respectively. ${ }^{41-45,52}$ As the quantity of $0.1 \mathrm{M}$ concentrated AuNPs increases from 200 to $1000 \mathrm{ng} \mathrm{mL} \mathrm{mL}^{-1}$, the cell inhibition percentage also increases gradually. This is happened due to insertion and penetration of more number of ideal sized $(\sim 10$ $\mathrm{nm}$ ) AuNPs inside the cell through the permeability of cell membrane.

\subsection{Cell imaging}

The laser scanning confocal microscopic bright field images of K562 cells illustrate the morphology of cells. The cells before (image (a) of Fig. 8) treatment shows clear spherical shaped morphology and tighten cell membrane whereas bright field image of K562 cells (image (b) of Fig. 8) after treatment with synthesized AuNPs shows ruptured and completely disrupted morphology and cell membrane with swelled and fragmented cellular organelles.

The morphology of K562 cells treated with the synthesized ideal sized $(\sim 10 \mathrm{~nm})$ and $\sim 40 \mathrm{~nm}$ AuNPs has been evaluated through the laser scanning confocal microscopy (LSCM) imaging. The $1000 \mathrm{ng} \mathrm{mL} \mathrm{m}^{-1}$ of AuNPs shows significant cell inhibition i.e. $88 \%$, which is ever reported inhibition for such $\sim 10 \mathrm{~nm}$ sized AuNPs. Similar method has been followed for the cell inhibition measurements for large sized ( $\sim 40 \mathrm{~nm})$ AuNPs. The Fig. $66 \dagger$ bar graph illustrates the cell inhibition (\%) with large sized $(\sim 40 \mathrm{~nm})$ AuNPs. The synthesized $\sim 40 \mathrm{~nm}$ sized gold nanoparticles were directly incubated with the cultured K562 cells and inhibition measurements performed after $36 \mathrm{~h}$ treatment and found $76 \%$ cell inhibition for $1000 \mathrm{ng} \mathrm{mL}^{-1}$ quantity of $0.1 \mathrm{M}$ concentrated AuNPs. ${ }^{44-47,53}$
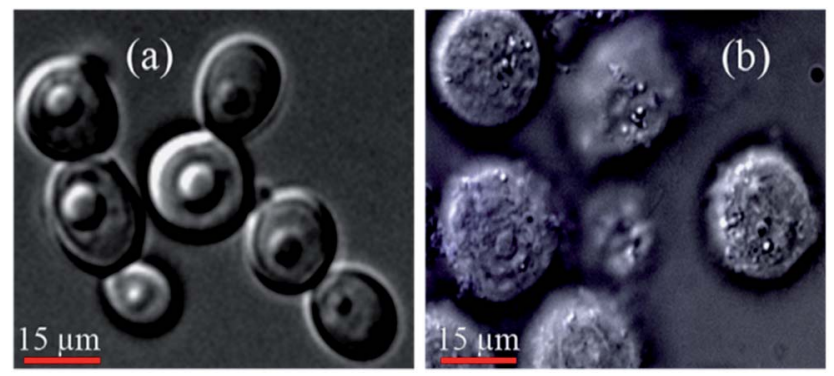

Fig. 8 Laser scanning confocal microscopic images of K562 cells illustrate the morphology of cells (a) before treatment which means cells as cultured and (b) cells after treatment with synthesized AuNPs.
There is around $12 \%$ cell inhibition difference for $\sim 10 \mathrm{~nm}$ sized AuNPs to $\sim 40 \mathrm{~nm}$ sized AuNPs and this is happened because of the greater penetration of the $\sim 10 \mathrm{~nm}$ sized AuNPs inside the K562 cells. From this cell inhibition studies, it is confirmed that the larger nanoparticle will not easily enter inside the cell membrane. The ideal sized metal (Au) nanoparticles shows significant permeability followed by the potential cell inhibition efficacy. The Fig. 9ad corresponds to confocal microscopic images of K562 cells treated with smaller sized (avg. particle size is $10.4 \mathrm{~nm}$ ) AuNPs. The cellular morphology for treated cells has been stained with different fluorescent dyes for better understating of size and shape of cells. The image (a) is for K562 cell stained with green fluorescent dye FITC (fluorescent isothiocyanate), image (b) corresponds to cell stained with red fluorescent Rho-6G, ${ }^{\mathbf{4 8 - 5 1 , 5 5}}$ image (c) corroborates the internal cellular materials stained with blue fluorescent dye DAPI and image (d) corresponds to merge of all the fluorescent images $(\mathrm{a}-\mathrm{c})$. The Fig. $\mathrm{S} 7$ and $\mathrm{S} 8 \dagger$ also corroborate the morphology of K562 cells treated with the ideal sized AuNPs. From these figures, the ruptured morphology of K562 cells has been depicted. Basically, healthy and as cultured K562 cells are spherical in shape without any structural irregularities and distortions. The swelling and shrinkage in cell morphology deliberates the ruptured morphology of cells treated with synthesized AuNPs. Similarly, the Fig. 10a-d and $\mathrm{S} 9 \mathrm{a}-\mathrm{d} \dagger$ corresponds to confocal
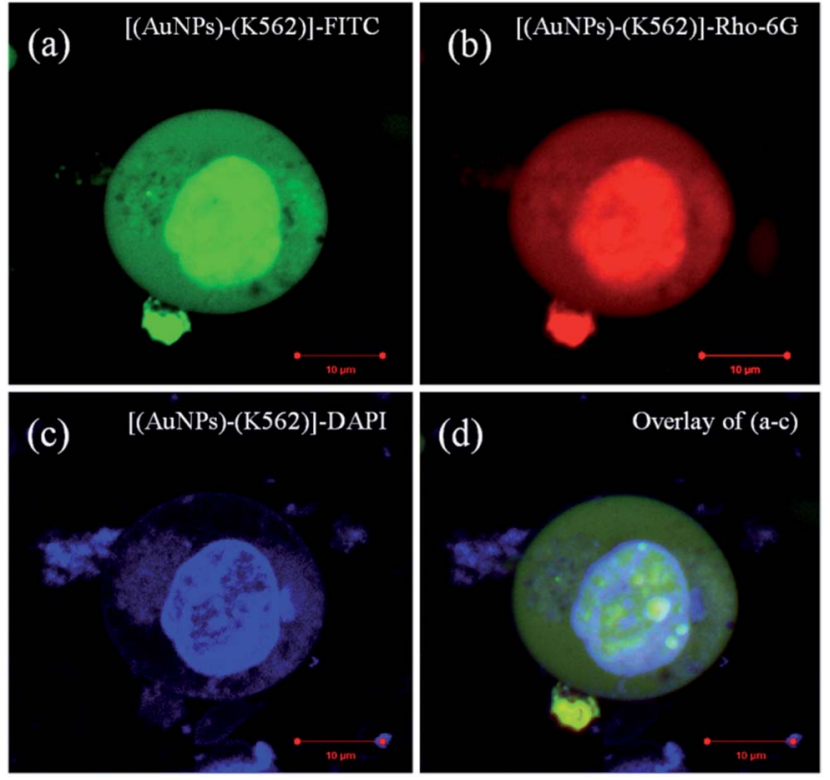

Fig. 9 Confocal microscopic images of $\mathrm{K} 562$ cells treated with smaller (avg. particle size is $10.4 \mathrm{~nm}$ ) AuNPs. The cellular morphology for treated cells has been stained with different fluorescent dyes for better understating of size and shape of cells. The image (a) for K562 cell stained with green fluorescent dye FITC (fluorescent isothiocyanate), image (b) corresponds to cell stained with red fluorescent Rho-6G, ${ }^{48,54}$ image (c) corroborates the internal cellular materials stained with blue fluorescent dye DAPI and image (d) corresponds to merge of all the fluorescent images $(a-c)$. 

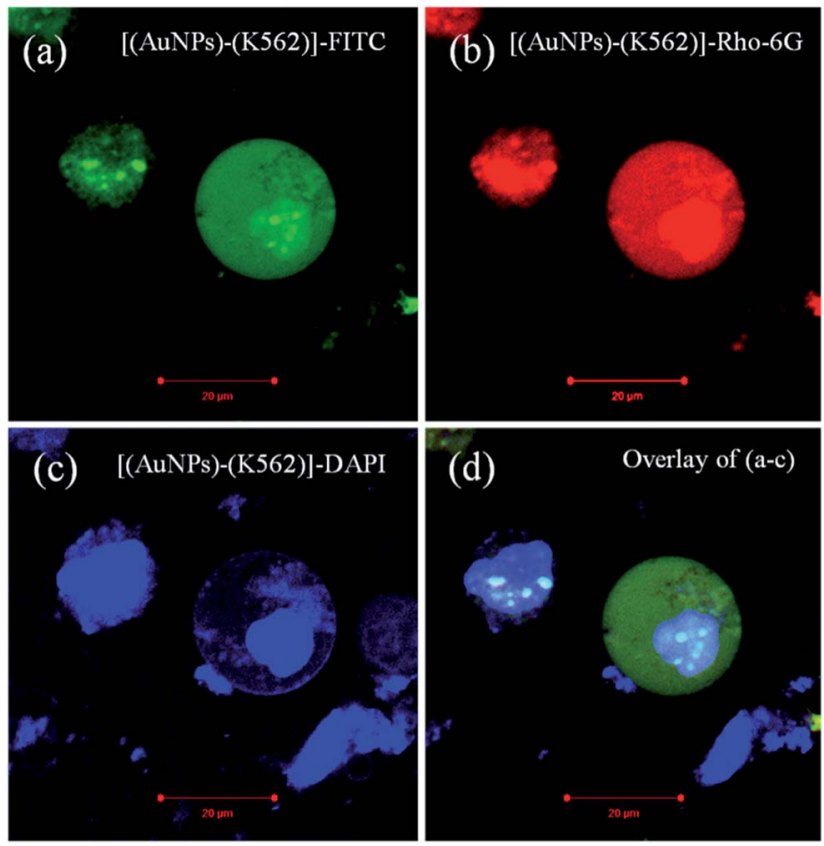

Fig. 10 Confocal microscopic images of K562 cells treated with large sized (avg. particle size is $40.2 \mathrm{~nm}$ ) AuNPs. The cellular morphology for treated cells has been stained with different fluorescent dyes for better understating of size and shape of cells. The image (a) for K562 cell stained with green fluorescent dye FITC (fluorescent isothiocyanate), image (b) corresponds to cell stained with red fluorescent Rho-6G, image (c) corroborates the internal cellular materials stained with blue fluorescent dye DAPI and image $(d)$ corresponds to merge of all the fluorescent images $(a-c)$.

microscopic images of K562 cells treated with large sized (avg. particle size is $40.2 \mathrm{~nm}$ ) AuNPs. From the Fig. S9a-d, $\dagger$ the mitochondria shaped K562 cell can be observed at higher magnifications which is structurally distorted after $36 \mathrm{~h}$ treatment with synthesized $\sim 40 \quad \mathrm{~nm}$ sized AuNPs. ${ }^{11,17,19,43,49,51,56,57}$

\section{Conclusions}

Herein, the solvent effect on the synthesis of two different sized ( $\sim 10 \mathrm{~nm}$ and $\sim 40 \mathrm{~nm}$ ) AuNPs has been reported. The affect of polar organic solvent DMF with respect to reducing agent $\mathrm{NaBH}_{4}$ gives nanoparticle with an avg. size of $10.4 \mathrm{~nm}$. It has been denoted as the $\left[(\mathrm{DMF})-\left(\mathrm{NaBH}_{4}\right) \rightarrow\right.$ (avg. size $\left.\left.10.4 \mathrm{~nm}\right)\right]$. Similar synthesis method has been followed with NMPL and $\mathrm{Na}_{3} \mathrm{C}_{6} \mathrm{H}_{5} \mathrm{O}_{7}$ and obtained particles with an avg. size of $40.2 \mathrm{~nm}$. It has been denoted as the $\left[(\mathrm{NMPL})-\left(\mathrm{Na}_{3} \mathrm{C}_{6} \mathrm{H}_{5} \mathrm{O}_{7}\right) \rightarrow\right.$ (avg. size $\left.\left.40.2 \mathrm{~nm}\right)\right]$. The AuNPs size, shape and morphology have been confirmed through the TEM and HRTEM characterizations followed by the SAED patterns. These nanoscale AuNPs were used to evaluate the working efficacy on K562 (leukaemia) blood cancer cells. An in vitro cell based studies were performed to examine the potential cell inhibition of synthesized AuNPs and $\sim 10 \mathrm{~nm}$ sized AuNPs gives significant inhibition i.e. $88 \%$ which is ever reported with such ideal nanosized particles. This $\sim 10 \mathrm{~nm}$ sized AuNPs are recommended for the treatment of breast, lung, and liver cancers and other theranostic applications.

\section{Conflicts of interest}

There are no conflicts to declare.

\section{Acknowledgements}

The authors would like to acknowledge UGC for financial assistance under the RGNF Scheme. The Centre for Nanotechnology, University of Hyderabad for TEM and HR-TEM characterizations. The MLR Institute of Technology, Hyderabad for financial assistance and facilities.

\section{Notes and references}

1 C. Amgotha, S. J. D. S. Doddapanenib, G. Dharmapuric and M. Lakavathu, Mater. Sci. Eng. C, 2018, 92, 790.

2 Y. J. Kim, S. Ryou, S. Kim, J. Yeom, M. S. Han, K. Lee and M. Seong, J. Mater. Chem., 2012, 22, 25036.

3 L. Li, M. Wanga, Y. Chen and S. Jiang, J. Colloid Interface Sci., 2012, 387, 146.

4 T. Chen, S. Xu, T. Zhao, L. Zhu, D. Wei, Y. Li, H. Zhang and C. Zhao, ACS Appl. Mater. Interfaces, 2012, 4, 5766.

5 E. C. Dreaden, A. M. Alkilany, X. Huang, C. J. Murphy and M. A. El-Sayed, Chem. Soc. Rev., 2012, 41, 2740.

6 A. Kumar, B. M. Boruah and X. Liang, J. Nanomater., 2011, 2011, 1 .

7 Q. Zhang, N. Large and H. Wang, ACS Appl. Mater. Interfaces, 2014, 6, 17255.

8 C. Amgoth, G. Dharmapuri, A. M Kalle and P. Paik, Nanotechnology, 2016, 27, 125101.

9 A. Singh, A. Guleria, S. Neogy and M. C. Rath, Arabian J. Chem., 2018, DOI: 10.1016/j.arabjc.2018.09.006.

10 M. Garcia, M. P. Beecham, K. Kempe, D. M. Haddleton, A. Khan and A. Marsh, Eur. Polym. J., 2015, 66, 444.

11 C. Amgoth, M. Lakavathu and D. S. D. S. Joshi, Bull. Mater. Sci., 2018, 41, 142.

12 W. Lai, J. Zhuang, X. Que, L. Fu and D. Tang, Biomater. Sci., 2014, 2, 1073.

13 A. Baeza, E. Guisasola, E. Ruiz-Hernandez and M. ValletRegi, Chem. Mater., 2012, 24, 517.

14 E. M. Perassi, C. Hrelescu, A. Wisnet, M. Doblinger, C. Scheu, F. Jackel, E. A. Coronado and J. Feldmann, ACS Nano, 2014, 8, 4395.

15 N. Chaubey, A. K. Sahoo, A. Chattopadhyay and S. S. Ghosh, Biomater. Sci., 2014, 2, 1080.

16 Z. Liang, X. Li, Y. Xie and S. Liu, Biomed. Mater., 2014, 9, 025012.

17 R. Gunti, G. Dharmapuri, S. J. Doddapaneni and C. Amgoth, Adv. Mater. Lett., 2017, 8, 1057.

18 C. Yang, M. Bian and Z. Yang, Biomater. Sci., 2014, 2, 651.

19 C. Amgoth and S. Joshi, Mater. Res. Express, 2017, 4, 105306.

20 A. H. R. Koch, G. Leveque, S. Harms, K. Jaskiewicz, M. Bernhardt, A. Henkel, C. Sonnichsen, K. Landfester and G. Fytas, Nano Lett., 2014, 7, 41. 
21 R. M. Talom, G. Fuks, C. Mingotaud, S. Gineste and F. Gauffre, J. Colloid Interface Sci., 2012, 387, 180.

22 C. Amgotha and G. Dharmapuri, Mater. Today: Proc., 2016, 3, 3833.

23 A. Ganguly, O. Trovato, S. Duraisamy, J. Benson, Y. Han, C. Satriano and P. Papakonstantinou, J. Phys. Chem. C, 2019, DOI: 10.1021/acs.jpcc.9b00303.

24 R. Gupta and B. Rai, Sci. Rep., 2017, 7, 45292.

25 X. Wu, Y. Tian, M. Yu, J. Hanb and S. Han, Biomater. Sci., 2014, 2, 972.

26 W. Li, X. Zhu, J. Wang, R. Liang, J. Li, S. Liu, G. Tu and J. Zhu, J. Colloid Interface Sci., 2014, 418, 81.

27 J. Hove, L. M. I. Schijven, J. Wang and A. H. Velders, Chem. Commun., 2018, 54, 13355.

28 L. Liu, X. Zhang and J. Chaudhuri, Mater. Res. Express, 2014, 1, 035033.

29 Y. La, C. Park, T. J. Shin, S. H. Joo, S. Kang and K. T. Kim, Nat. Chem., 2014, 6, 534.

30 R. Balasubramanian, J. Xu, B. Kim, B. Sadtler and A. Wei, J. Dispersion Sci. Technol., 2001, 22, 485.

31 Q. Zhang, N. Large, P. Nordlander and H. Wang, J. Phys. Chem. Lett., 2014, 5, 370.

32 M. N. Martin, J. I. Basham, P. Chando and S. Eah, Langmuir, 2010, 26, 7410.

33 Z. Shervani and Y. Yamamoto, Mater. Lett., 2011, 65, 92.

34 A. Singh, A. Guleria, A. Kunwar, S. Neogy and M. C. Rath, Mater. Res. Express, 2019, 6, 015029.

35 Z. Chu, S. Zhang, C. Yin, G. Lin and Q. Li, Biomater. Sci., $2014,2,827$.

36 K. Letchford and H. Burt, Eur. J. Pharm. Biopharm., 2007, 65, 259.

37 H. Chen, X. Chi, B. Li, M. Zhang, Y. Ma, S. Achilefud and Y. Gu, Biomater. Sci., 2014, 2, 996.

38 D. Q. M. Craig, Int. J. Pharm., 2002, 231, 131.

39 S. Shen, X. Chen, X. Zhang, J. Miao and B. Zhao, J. Mater. Chem. B, 2015, 3, 919.

40 C. E. Bradburne, J. B. Delehanty, K. B. Gemmill, B. C. Mei, H. Mattoussi, K. Susumu, J. B. Blanco-Canosa,
P. E. Dawson and I. L. Medintz, Bioconjugate Chem., 2013, 24, 1570 .

41 J. Fan, F. Zeng, S. Wu and X. Wang, Biomacromolecules, 2012, 13, 4126.

42 Y. Yang, W. Wang, T. Chen and Z. Chen, ACS Appl. Mater. Interfaces, 2014, 6, 21468.

43 M. Hu, M. Chen, G. Li, Y. Pang, D. Wang, J. Wu, F. Qiu, X. Zhu and J. Sun, Biomacromolecules, 2012, 13, 3552.

44 K. J. Clear, K. M. Harmatys, D. R. Rice, W. R. Wolter, M. A. Suckow, Y. Wang, M. Rusckowski and B. D. Smith, Bioconjugate Chem., 2015, 27, 363.

45 A. Singh, A. Kunwar and M. C. Rath, J. Nanosci. Nanotechnol., 2018, 18, 3419.

46 A. Singh, A. Guleria, A. Kunwar, S. Neogy and M. C. Rath, Mater. Chem. Phys., 2017, 199, 609.

47 S. Shukla, C. Dickmeis, A. S. Nagarajan, R. Fischer, U. Commandeur and N. F. Steinmetz, Biomater. Sci., 2014, 2, 784 .

48 P. Abinayasri, M. Nageshwari, B. Meenarathi and R. Anbanbarasan, Bull. Mater. Sci., 2017, 40, 591.

49 W. Feng, X. Zhou, C. He, K. Qiu, W. Nie, L. Chen, H. Wang, X. Mo and Y. Zhang, J. Mater. Chem. B, 2013, 1, 5886.

50 Y. Jin Kim, S. Ryou, S. Kim, J. Yeom, M. Han, K. Lee and M. Seong, J. Mater. Chem., 2012, 22, 25036.

51 Q. Zhang, J. Zhu, L. Song, J. Zhang, D. Kong, Y. Zhao and Z. Wang, J. Mater. Chem. B, 2013, 1, 6402.

52 Q. Mu, G. Jiang, L. Chen, H. Zhou, D. Fourches, A. Tropsha and B. Yan, Chem. Rev., 2014, 114, 7740-7781.

53 Y. Huang, Q. Liu, Y. Wang, N. He, R. Zhao, J. Choo and L. Chen, Nanoscale, 2019, 11, 12220-12229.

54 Q. Yu, Y. Wang, R. Mei, Y. Yin, J. You and L. Chen, Anal. Chem., 2019, 91, 5270-5277.

55 R. Mei, Y. Wang, W. Liu and L. Chen, ACS Appl. Mater. Interfaces, 2019, 10, 23606-23616.

56 W. Zhang, Y. Wang, X. Sun, W. Wang and L. Chen, Nanoscale, 2014, 6, 14514.

57 H. Park, S. Lee, L. Chen, E. K. Lee, S. Y. Shin, Y. H. Lee, S. W. Son, C. H. Oh, J. M. Song, S. H. Kang and J. Choo, Phys. Chem. Chem. Phys., 2009, 11, 7444-7449. 